\title{
Hawking radiation from extremal and nonextremal black holes
}

\author{
R. Balbinot, ${ }^{1, *}$ A. Fabbri, ${ }^{2, \dagger}$ S. Farese, ${ }^{2, *}$ and R. Parentani ${ }^{3, \S}$ \\ ${ }^{1}$ Dipartimento di Fisica dell'Università di Bologna and INFN sezione di Bologna, 40126 Bologna, Italy \\ ${ }^{2}$ Departamento de Física Teórica and IFIC, Centro Mixto Universidad de Valencia-CSIC. \\ Facultad de Física, Universidad de Valencia, 46100, Burjassot, Valencia, Spain \\ ${ }^{3}$ Laboratoire de Physique Théorique, CNRS UMR 8627, Bât. 210, Université Paris XI, 91405 Orsay Cedex, France
}

(Received 2 October 2007; published 7 December 2007)

\begin{abstract}
The relationship between Hawking radiation emitted by nonextremal and extremal Reissner-Nordström black holes is critically analyzed. A careful study of a series of regular collapsing geometries reveals that the stress energy tensor stays regular in the extremal limit and is smoothly connected to that of nonextremal black holes. The unexpected feature is that the late time transients which played little role in the nonextremal case are necessary to preserve the well-defined character of the flux in the extremal case. The known singular behavior of the static energy density of extremal black holes is recovered from our series by neglecting these transients, when performing what turns out to be an illegitimate late time limit. Although our results are derived in two-dimensional settings, we explain why they should also apply to higher dimensional black holes.
\end{abstract}

DOI: 10.1103/PhysRevD.76.124010

PACS numbers: 04.62.+v, 04.70.Dy

\section{INTRODUCTION}

Nonextremal Reissner-Nordström black holes (NEBH) form a two parameter family in which the inequality $M>$ $|Q|$ holds, where $M$ and $Q$ are their mass and their charge, respectively. Extreme Reissner-Nordström black holes (EBH) form a one parameter family, and obey $M=|Q|$. Their surface gravity vanishes and so does their Hawking temperature. Therefore, if discharge does not occur, EBH can be regarded as the end point configuration of the evaporation of NEBH.

However, EBH and NEBH seem rather disjoint in many aspects. On one hand, the Euclidean section of EBH is very different than that of NEBH. It possesses an infinite throat, the horizon sitting at the end of it. Since the geometry is regular when approaching the throat, the period of Euclidean time is arbitrary, unlike for NEBH where the period must be $2 \pi / \kappa$, where $\kappa$ is their surface gravity, in order for not having a conical singularity on the horizon. This has led some authors [1-5] to conjecture that the Bekenstein-Hawking area-entropy relation does not apply to EBH which should be characterized by zero entropy. Since then, this conjecture has been invalidated by string theory which confirmed the Bekenstein-Hawking formula by counting microstates of some particular EBH [6].

On the other hand, using the more familiar settings of quantum field theory in curved space, EBH seem to be plagued by divergences of the stress tensor which are absent for NEBH, thereby reinforcing the idea that EBH should be considered as forming a disconnected family. With more details, when considering the formation of a

\footnotetext{
*balbinot@bo.infn.it

†afabbri@ific.uv.es

\$Sara.Farese@uv.es

§arenta@th.u-psud.fr
}

NEBH by gravitational collapse, any regular state evolves at late time to a stationary state, often referred to as the "Unruh" vacuum, which is characterized by the condition of no incoming flux and by the regularity on the future horizon (as seen in a freely falling frame). This regularity is ensured by the steady thermal radiation with Hawking temperature $\kappa / 2 \pi$. When taking the extremal limit of this stationary situation, that is $M \rightarrow|Q|$, the outgoing flux disappears since the surface gravity vanishes, but the resulting stress tensor is found to be singular [7-9], in a way similar to what is obtained in the "Boulware" vacuum of NEBH which is the stationary state with no Hawking radiation.

However, it has been also shown [10,11] that when one considers directly the formation of an EBH (for example by the collapse of a shell with $M=|Q|$ ), the resulting stress tensor is regular on the future horizon, in agreement with the general analysis of [12]. This result seems to contradict what we just obtained by considering first the formation of a NEBH and then taking the extremal limit since a singular stress tensor was found. Were this contradiction to persist, this would establish the fact that NEBH and $\mathrm{EBH}$ are indeed quantum mechanically distinct objects since one could not obtain expectation values for EBH from those evaluated with NEBH. Such conclusion was reached in [13] where it is claimed that the extremal case "in no sense represents a limit of the nonextremal case but implies a real discontinuity."

The purpose of this paper is to show that this conclusion is not correct. By a careful analysis of the extremal limit, we shall demonstrate the continuity of the expectation values in the limit $M \rightarrow|Q|$. To have a well-defined limiting procedure, we shall consider a series of regular collapsing geometries with $M \rightarrow|Q|$, and compute the local fluxes for every value of $(M, Q)$. Two subtle points are encountered in this limit. First, the regularity is preserved 
in the extremal only by taking into account the late time transients which played no significant role for NEBH. Moreover, the properties of these transients are independent of the collapse. Second, the late time limit (giving rise to stationary fluxes) cannot be taken before the extremal limit. This demonstrates that stationarity cannot be assumed when analyzing EBH, at least when dealing with regular collapsing geometries, as opposed to singular (and hence ill-defined) eternal configurations.

The plan of the paper is the following. In Sec. II we compute the stress tensor resulting from the collapse to a NEBH. The material presented in this section is rather standard, but with a special attention on transients in order to prepare taking the extremal limit. In Sec. III the analysis is repeated with the formation of an extremal $\mathrm{BH}$ and the necessity of keeping the transients is established. Then the smooth connection with the results of the previous section is demonstrated. Sec. IV contains the conclusions. Throughout the paper we shall work with a twodimensional analytical treatment, and at the end of the paper we argue that our results should also apply to fourdimensional BH. An appendix contains the expressions of the stress tensor of $2 \mathrm{D}$ massless fields we use in the text.

\section{HAWKING RADIATION EMITTED BY NEBH}

As shown by Hawking [14], the formation of a $\mathrm{BH}$ triggers a vacuum instability resulting in the emission of particles radiated towards infinity. When the $\mathrm{BH}$ is nonextremal, at late time and independently on the details of the collapse (besides its regularity [15]), one obtains a stationary flow of thermal radiation with Hawking temperature $T_{H}=\kappa / 2 \pi$, where $\kappa$ is the surface gravity of the outer horizon. The key properties of the associated stress tensor can be thus obtained by considering the formation of a $\mathrm{BH}$ by the collapse of a spherically symmetric ingoing null thin shell. Indeed, the key property to get Hawking radiation is the regularity of the geometry which is guaranteed when the infalling trajectory is inertial. As shown in [16], it suffices that the trajectory be nonsingular across the future horizon.

In this section, we consider the nonextremal case, i.e. $M>|Q|$. We consider the collapse of an ingoing charged null shell located at $v=v_{0}$. For $v<v_{0}$ the space-time is Minkowski and the metric reads (dropping the angular variables)

$$
d s^{2}=-d u_{\text {in }} d v
$$

where

$$
u_{\text {in }}=t_{\text {in }}-r, \quad v=t_{\text {in }}+r .
$$

Outside the shell, for $v>v_{0}$, one has

$$
d s^{2}=-f(r) d u d v=-\left(1-\frac{2 M}{r}+\frac{Q^{2}}{r^{2}}\right) d u d v,
$$

where now

$$
u=t-r^{*}, \quad v=t+r^{*},
$$

$r^{*}$ being the tortoise radial coordinate

$$
\begin{aligned}
r^{*}(r ; M, Q)= & \int^{r} \frac{d r^{\prime}}{1-\frac{2 M}{r^{\prime}}+\frac{Q^{2}}{r^{2}}} \\
= & r+\frac{1}{2 \kappa_{+}} \ln \left[\kappa_{+}\left(r-r_{+}\right)\right] \\
& -\frac{1}{2 \kappa_{-}} \ln \left[\kappa_{-}\left(r-r_{-}\right)\right],
\end{aligned}
$$

where $\kappa_{ \pm}$and $r_{ \pm}$are the surface gravities and the radii of the two horizons (outer and inner, respectively)

$$
\kappa_{ \pm}=\frac{\sqrt{M^{2}-Q^{2}}}{r_{ \pm}^{2}}, \quad r_{ \pm}=M \pm \sqrt{M^{2}-Q^{2}} .
$$

Asymptotic flatness implies that the ingoing null coordinate $v$ is the same on both sides of the shell. On the other hand the relation between $u_{\text {in }}$ and $u$ can be found by requiring the continuity of the radial coordinate $r$ along the shell. From Eqs. (2) and (4) evaluated on the shell we have

$$
\frac{v_{0}-u_{\text {in }}}{2}=r, \quad \frac{v_{0}-u}{2}=r^{*} .
$$

Using Eq. (5) and eliminating $r$ between the above two equations we exactly get

$$
\begin{aligned}
u= & u_{\text {in }}-\frac{1}{\kappa_{+}} \ln \left[\kappa_{+}\left(v_{0}-u_{\text {in }}-2 r_{+}\right)\right] \\
& +\frac{1}{\kappa_{-}} \ln \left[\kappa_{-}\left(v_{0}-u_{\text {in }}-2 r_{-}\right)\right] .
\end{aligned}
$$

From this we see that the event horizon, defined by $u=$ $+\infty$, corresponds to $u_{\text {in }}=v_{0}-2 r_{+}$and to $r=r_{+}$. To simplify the forthcoming equation, we introduce a new null coordinate

$$
U_{\text {in }}=u_{\text {in }}-v_{0}+2 r_{+},
$$

which vanishes on the event horizon and which is linearly related to $u_{\text {in }}$. We also notice that in the late time limit, $u \rightarrow$ $\infty$, Eq. (8) yields

$$
u=-\frac{1}{\kappa_{+}} \ln \left(-\kappa_{+} U_{\text {in }}\right)+D+O\left(U_{\text {in }}\right),
$$

where $D$ is a constant which plays no role as it can be absorbed in $u$. When ignoring the linear correction $O\left(U_{\text {in }}\right)$ we recover the usual relation between the Kruskal coordinate $U_{K}$ and the asymptotic coordinate $u$ :

$$
u=-\frac{1}{\kappa_{+}} \ln \left(-\kappa_{+} U_{K}\right) .
$$

This relation could be obtained by considering the eternal $\mathrm{BH}$ geometry, i.e. without referring to any collapse. As we shall see, the important physical consequence of the late 
time correspondence between $U_{\text {in }}$ and $U_{K}$ is that the initial vacuum (containing no negative frequency with respect to $u_{\text {in }}$ or $U_{\text {in }}$ ) will rapidly evolve into the Unruh vacuum (the state containing no negative frequency with respect to $U_{K}$ ), i.e. the transient flux will rapidly die out. The decay of these transients is governed by the difference between $U_{K}$ and $U_{\text {in }}$. Near the horizon, they are related by

$$
U_{\text {in }}=D^{\prime} U_{K}+O\left(U_{K}^{2}\right),
$$

where $D^{\prime}$ is another irrelevant constant.

We now consider a massless minimally coupled scalar field propagating in the above collapsing geometry. Taking the quantum state of the field (|in〉) to be Minkowski vacuum on $I^{-}$implies that the state is vacuum with respect to the positive frequency modes

$$
\phi_{\omega}^{\mathrm{in}}(v) \propto e^{-i \omega v}, \quad \phi_{\omega}^{\mathrm{in}}(u) \propto e^{-i \omega U_{\text {in }}(u)} .
$$

As recalled in the appendix, this determines the expectation values of the stress tensor everywhere. Inside the shell (i.e. $v<v_{0}$ ), we have

$$
\left\langle\text { in }\left|T_{\mu \nu}\right| \text { in }\right\rangle \equiv 0,
$$

because the geometry is flat. Outside the shell, in the BH geometry, the stress tensor splits into a static part, which is completely determined by $f(r)=1-2 M / r+Q^{2} / r^{2}$ and which can be viewed as a vacuum polarization, and a timedependent outgoing flux which is caused by the collapse:

$$
\begin{aligned}
& \left\langle\text { in }\left|T_{u v}\right| \text { in }\right\rangle=-\frac{1}{24 \pi}\left(1-\frac{2 M}{r}+\frac{Q^{2}}{r^{2}}\right)\left(\frac{M}{r^{3}}-\frac{3}{2} \frac{Q^{2}}{r^{4}}\right), \\
& \left\langle\text { in }\left|T_{v v}\right| \text { in }\right\rangle=\frac{1}{24 \pi}\left(-\frac{M}{r^{3}}+\frac{3}{2} \frac{M^{2}+Q^{2}}{r^{4}}-\frac{3 M Q^{2}}{r^{5}}+\frac{Q^{4}}{r^{6}}\right),
\end{aligned}
$$

$$
\left\langle\text { in }\left|T_{u u}\right| \text { in }\right\rangle=\left\langle\text { in }\left|T_{v v}(r)\right| \text { in }\right\rangle-\frac{1}{24 \pi}\left\{u_{\mathrm{in}}, u\right\}(u) .
$$

The outgoing flux is governed by the Schwarzian derivative $\left\{u_{\text {in }}, u\right\}$. Using Eqs. (8) and (9), we get

$$
\begin{aligned}
& \left\{u_{\text {in }}, u\right\}=\left\{U_{\text {in }}, u\right\} \\
& =-2 \kappa_{+}^{2} \frac{\left[1-\frac{\kappa_{+}}{\kappa_{-}} \frac{U_{\text {in }}^{3}}{\left(U_{\text {in }}-2\left(r_{+}-r_{-}\right)\right)^{3}}\right]}{\left[1-\kappa_{+} U_{\text {in }}-\frac{\kappa_{+}}{\kappa_{-}} \frac{U_{\text {in }}}{\left(U_{\text {in }}-2\left(r_{+}-r_{-}\right)\right)}\right]^{3}}
\end{aligned}
$$

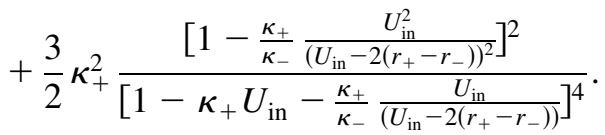

At early times, when the shell radius is much larger than $r_{+}, u \sim U_{\text {in }} \rightarrow-\infty$ and the flux vanishes as one might expect. At late times, for $u \rightarrow+\infty$ and $U_{\text {in }} \rightarrow 0$, we obtain

$$
\left\{U_{\mathrm{in}}, u\right\}^{u=+\infty}-\frac{\kappa_{+}^{2}}{2}+C^{\prime} U_{\mathrm{in}}^{2}
$$

The constant term describes the stationary Hawking flux at the temperature $T_{H}=\kappa_{+} / 2 \pi$. It depends only on the final geometry, and is thus independent on the choice of the collapsing configuration. Indeed, it coincides with the flux calculated in the Unruh vacuum, the stationary state where outgoing modes are positive frequency with respect to the Kruskal coordinate $U_{K}$ of Eq. (11). This directly follows from

$$
\left\{U_{K}, u\right\}=-\kappa_{+}^{2} / 2 .
$$

From Eq. (19), we also learn that the transient terms, which depend on the details of the collapse, die out with two powers of $U_{\text {in }}$, i.e. like $\exp \left(-2 \kappa_{+} u\right)$ as $u \rightarrow+\infty$ in terms of the asymptotic null time, and not only with one power as one might have expected. In brief, at late times, the outgoing flux becomes stationary and given by Eq. (17) with $\left\{u_{\mathrm{in}}, u\right\}$ given by the first term of Eq. (19).

The crucial property of the expectation values (15)-(17) is their regularity on the future outer horizon. We remind the reader that regularity on the future horizon requires that the energy density measured by a free falling observer

$$
\rho_{F F} \equiv T_{\mu \nu} \frac{d x^{\mu}}{d \tau} \frac{d x^{\nu}}{d \tau}
$$

is finite. In the above $\tau$ is the proper time of the observer. In the limit $r \rightarrow r_{+}, \frac{d v}{d \tau}$ is constant and $\frac{d u}{d \tau} \sim \frac{1}{f}$. The finiteness of $\rho_{F F}$ thus leads to the following conditions [17]:

$$
\begin{gathered}
\lim _{r \rightarrow r_{+}} f^{-1}\left\langle T_{u v}\right\rangle<\infty, \\
\lim _{r \rightarrow r_{+}}\left\langle T_{v v}\right\rangle<\infty, \\
\lim _{r \rightarrow r_{+}} f^{-2}\left\langle T_{u u}\right\rangle<\infty,
\end{gathered}
$$

where $f \rightarrow\left(r-r_{+}\right)\left(r_{+}-r_{-}\right) / r_{+}^{2}$. The first two conditions are satisfied since $\left\langle T_{u v}\right\rangle$ is state independent and vanishes linearly as $r-r_{+}$and since $\left\langle T_{v v}\right\rangle$ is regular in the in vacuum. The last one requires more care. From Eqs. (20), (A5), and (A8) we obtain that the late time limit (Unruh vacuum) behaves as

$$
\begin{aligned}
\left\langle U\left|T_{u u}\right| U\right\rangle & =\left\langle\text { in }\left|T_{v v}\right| \text { in }\right\rangle+\frac{1}{48 \pi} \kappa_{+}^{2} \\
& =-\frac{1}{192 \pi}\left(f^{\prime}(r)^{2}-2 f(r) f^{\prime \prime}(r)-f^{\prime}\left(r_{+}\right)^{2}\right),
\end{aligned}
$$

where we have used $\kappa_{+}=f^{\prime}\left(r_{+}\right) / 2$. In the limit $r \rightarrow r_{+}$, we obtain

$$
\left\langle U\left|T_{u u}\right| U\right\rangle^{r \rightarrow r^{+}} f^{2} C,
$$

where $C=\frac{f^{\prime}\left(r_{+}\right) f^{\prime \prime \prime}\left(r_{+}\right) r_{+}^{4}}{192 \pi\left(r_{+}-r_{-}\right)^{2}}$ is a constant depending on $M$ and $Q$. The steady part of the outgoing flux in the in vacuum is thus regular on the horizon [18]. 
It is equally important to notice that the transients which have been neglected above do not spoil this regularity because they decrease with two powers of $r-r_{+}$as $r \rightarrow$ $r_{+}$, see Eq. (19). (We remind the reader that in a freely falling frame the following relations hold across the horizon: $d \tau \propto d U_{\text {in }} \propto-d r$ where $\tau$ is the proper time in this frame.) These two powers compensate the divergence of $1 / f^{2} \sim 1 /\left(r-r_{+}\right)^{2}$ in Eq. (24). Thus the transient contribution, taken alone, is regular on the horizon.

In conclusion, we have verified that the regularity condition applied to the outgoing flux is satisfied on the outer future horizon of a NEBH both by the steady Unruh expectation values and by the late time transients.

\section{HAWKING RADIATION EMITTED BY EBH}

\section{A. The stationary expectation values}

Extremal $\mathrm{BH}$ are characterized by $M=|Q|$ and their line element can be obtained from Eq. (3) by taking the limit $M \rightarrow Q$. One gets

$$
d s^{2}=-\left(1-\frac{M}{r}\right)^{2} d u d v,
$$

where $u=t-r^{*}, v=t+r^{*}$ as before, and where the "extremal" tortoise coordinate is

$$
\begin{aligned}
r^{*}(r ; M) & =\int^{r} \frac{d r^{\prime}}{\left(1-M / r^{\prime}\right)^{2}} \\
& =r+M\left[\frac{-1}{r / M-1}+2 \ln (r / M-1)\right] .
\end{aligned}
$$

The novelty is that the merging of the two horizons causes a double zero of the metric $f=(1-M / r)^{2}$ on the horizon at $r=M$. As a consequence, the surface gravity vanishes, as can be seen by taking the limit $M \rightarrow Q$ in Eq. (6), and, equally important, $r^{*}$ now diverges as $-1 /(r-M)$ when approaching the horizon and no longer as a logarithm in $r-r_{+}$as was the case for NEBH in Eq. (5).

To obtain the stationary value of the stress tensor for $\mathrm{EBH}$, two approaches give the same result. The first one consists in performing the extremal limit $\kappa_{+} \rightarrow 0$ of the late time limit of Eqs. (15)-(17). The second one consists in working directly with the extremal metric given above, requiring that the stress tensor be static and vanish at infinity. In the second approach, the trace anomaly determines unambiguously the following expressions:

$$
\begin{gathered}
\left\langle T_{u v}\right\rangle_{\text {stat }}=-\frac{1}{24 \pi} \frac{M}{r^{3}}\left(1-\frac{3}{2} \frac{M}{r}\right)\left(1-\frac{M}{r}\right), \\
\left\langle T_{v v}\right\rangle_{\text {stat }}=-\frac{1}{24 \pi} \frac{M}{r^{3}}\left(1-\frac{M}{r}\right)^{3}, \\
\left\langle T_{u u}\right\rangle_{\text {stat }}=\left\langle T_{v v}\right\rangle_{\text {stat }} .
\end{gathered}
$$

The novelty is that Eq. (31) does not vanish sufficiently rapidly on the horizon to fulfill the regularity condition. In fact, since $f$ has a double zero, one gets

$$
\lim _{r \rightarrow M} f^{-2}\left\langle T_{u u}\right\rangle_{\text {stat }}=\lim _{r \rightarrow M}\left[-\frac{1}{24 \pi} \frac{M}{r^{3}}\left(1-\frac{M}{r}\right)^{-1}\right]=\infty .
$$

This implies that an observer free falling across the horizon will measure an infinite energy density. This fact, reinforced by the uniqueness of the stress tensor under the simple (and apparently sound) hypothesis of stationarity and asymptotic vanishing flux [19] has led to the conjecture that EBH might be singular objects from a quantum mechanical point of view [7-9]. In this case, they should be conceived as being disconnected from the regular NEBH (at least in two dimensions).

However, in $[10,11]$ it was shown that by considering the formation of a $\mathrm{BH}$ which is ab initio extremal, the resulting outgoing flux, $\left\langle\right.$ in $\left|T_{u u}\right|$ in $\rangle$, radically differs from Eq. (31). Namely, it is time dependent as one might have expected, but, more importantly, its late time dependence is univer$s a l$, i.e. independent of the regular collapse one has chosen, and such that $\left\langle\mathrm{in}\left|T_{u u}\right|\right.$ in $\rangle$ is regular on the horizon. One is therefore led to conclude that the above stationary expressions $\left\langle T_{a b}\right\rangle_{\text {stat }}$ do not characterize the late time behavior of the stress energy of regular EBH. To see how regularity is achieved, let us briefly review what happens when an EBH is formed by collapse.

\section{B. The flux emitted by an incipient EBH}

Consider the collapse of a charged null shell with $M=$ $Q$. In this case, repeating the steps of Sec. II, that is, using Eq. (28) in the place of Eq. (5), one finds

$$
\begin{aligned}
u & =u_{\text {in }}-4 M\left[\frac{-1}{2\left(\frac{v_{0}-u_{\text {in }}}{2 M}-1\right)}+\ln \left(\frac{v_{0}-u_{\text {in }}}{2 M}-1\right)\right] \\
& =U_{\text {in }}-4 M\left[\frac{M}{U_{\text {in }}}+\ln \left(-\frac{U_{\text {in }}}{2 M}\right)\right],
\end{aligned}
$$

where we have introduced the null coordinate

$$
U_{\text {in }}=u_{\text {in }}-v_{0}+2 M,
$$

which again vanishes on the future horizon. Evaluating the Schwarzian derivative $\left\{U_{\text {in }}, u\right\}$ one gets

$$
\begin{aligned}
\left\langle\text { in }\left|T_{u u}\right| \text { in }\right\rangle & =\left\langle T_{u u}\right\rangle_{\text {stat }}+\left\langle T_{u u}\right\rangle_{\text {transients }}, \\
& =\left\langle T_{u u}\right\rangle_{\text {stat }}-\frac{1}{24 \pi} \frac{8 M U_{\text {in }}^{3}}{\left(U_{\text {in }}-2 M\right)^{6}} .
\end{aligned}
$$

The last term describes the outgoing radiation. At early times, for $U_{\text {in }} \rightarrow-\infty$, it decreases with three powers of the affine null parameter $U_{\text {in }}$. At late times, for $u \rightarrow \infty$, it vanishes [20] as expected since there cannot be Hawking radiation, the surface gravity being zero. In terms of the asymptotic time $u$ the transient flux vanishes as $1 / u^{3}$, and not exponentially fast as transients died out for NEBH. 
Now let us examine the behavior of Eq. (35) when crossing the future horizon. We see that for $r \rightarrow M$ and $U_{\text {in }} \rightarrow 0$, the static vacuum polarization term and the transient flux both vanish with three powers of $r-M \propto-U_{\text {in }}$. Hence, if taken separately, both give a divergent contribution on the horizon, as shown in Eq. (32). However, when expressing $U_{\text {in }}$ as a function of $r-M$ along an arbitrary infalling geodesic, i.e. with $d v / d \tau=\lambda$ evaluated at the horizon characterizing the infalling velocity, one obtains

$$
U_{\text {in }}(r)=-2(r-M)+O\left((r-M)^{2}\right),
$$

where the first term is independent of $\lambda$. (This follows from the lightlike character of $d r$ on the horizon). This independence guarantees that the leading terms of the two contributions of Eq. (35) cancel each other:

$$
\begin{aligned}
\left\langle\text { in }\left|T_{u u}\right| \text { in }\right\rangle & =\left\langle T_{u u}\right\rangle_{\text {stat }}+\left\langle T_{u u}\right\rangle_{\text {transients }} \\
& =-\frac{(r-M)^{3}}{24 \pi M^{5}}-\frac{8 M U_{\text {in }}^{3}}{24 \pi(2 M)^{6}}+O\left((r-M)^{4}\right) \\
& =O\left((r-M)^{4}\right) .
\end{aligned}
$$

Hence $f^{-2}\left\langle\right.$ in $\left|T_{u u}\right|$ in $\rangle$ stays finite. The key point is that, even though the logarithmic term in Eq. (33) is subleading at late times, this term is necessary to get the above cancellation. Indeed, its omission would give a vanishing Schwarzian derivative and therefore would give back the singular behavior of $\left\langle T_{u u}\right\rangle_{\text {stat }}$ obtained in the former subsection. Notice that this term was omitted in [13], see Eq. (3.4), thereby leading to the erroneous conclusion that there is a "real discontinuity" between NEBH and EBH.

In brief, for EBH formed by the collapse of a shell, or more generally formed by a regular collapse [11], the stress tensor is in fact regular on the horizon. Having reached this conclusion, we finally arrive at the question we wanted to confront: why was this regular behavior missed in the extremal limit giving rise to Eq. (31)?

\section{The extremal limit of the nonextremal flux}

To answer the above question, one should reconsider how to implement the extremal limit. To this end, we first note that although the late time value of the Schwarzian derivative $\left\{U_{\text {in }}, u\right\}$ has a smooth limit for $\kappa_{+} \rightarrow 0$, as shown in Eqs. (18) and (19), thereby leading to the singular result of Eq. (32), the late time behavior of $u\left(U_{\mathrm{in}}\right)$, which is that of Eq. (11), has no well-defined limit $\kappa_{+} \rightarrow 0$. The illdefined character of this extremal limit tells us that the late time limit should not have been taken first.

So let us return to Eq. (8) which gives the exact relation between $U_{\text {in }}$ and $u$ and perform the extremal limit first. We notice that in terms of the surface gravities this limit reads $\kappa_{+} \rightarrow 0, \kappa_{-} \rightarrow 0, \kappa_{+} / \kappa_{-} \rightarrow 1$. So instead of using these as parameters, we shall reexpress the expressions directly in terms of $M$ and $Q$, and simply send $M \rightarrow Q$. We also notice that the nontrivial character of this limit entirely comes through the tortoise coordinate $r^{*}(r ; Q, M)$ which enters in the second equation of Eqs. (7). It is therefore sufficient to study the extremal limit of $r^{*}(r ; Q, M)$ of Eq. (5).

So, working at fixed $r$ (which amounts to not taking the late time limit), forming the half difference and the half sum of the two logarithms to sort out the singular and regular part, then using the values of $\kappa_{ \pm}$and $r_{ \pm}$of Eqs. (6), and only then performing the limit $M \rightarrow Q$, we successively get

$$
\begin{aligned}
2 r^{*}(r ; Q, M)-2 r & =\left(\frac{1}{2 \kappa_{+}}+\frac{1}{2 \kappa_{-}}\right) \ln \left|\frac{r-r_{+}}{r-r_{-}}\right|+\left(\frac{1}{2 \kappa_{+}}-\frac{1}{2 \kappa_{-}}\right) \ln \left|\frac{\left(r-r_{+}\right)\left(r-r_{-}\right)}{r_{+} r_{-}}\right| \\
& =\frac{2 M^{2}-Q^{2}}{\sqrt{M^{2}-Q^{2}}} \ln \left|1-\frac{2 \sqrt{M^{2}-Q^{2}}}{r-M+\sqrt{M^{2}}-Q^{2}}\right|+2 Q \ln \left|\frac{\left(r-r_{+}\right)\left(r-r_{-}\right)}{r_{+} r_{-}}\right| \\
& \stackrel{M \rightarrow Q}{\rightarrow} \frac{2 M^{2}-Q^{2}}{\sqrt{M^{2}-Q^{2}}} \times \frac{-2 \sqrt{M^{2}-Q^{2}}}{r-M+\sqrt{M^{2}-Q^{2}}}+4 Q \ln \frac{(r-Q)}{Q} \\
& \stackrel{M \rightarrow Q}{\rightarrow}-\frac{2 Q^{2}}{r-Q}+4 Q \ln \frac{(r-Q)}{Q},
\end{aligned}
$$

thereby recovering the behavior of the extremal tortoise coordinate one obtains from the extremal metric in Eq. (28). Therefore, when eliminating $r$ using the first of Eqs. (7) we also recover Eq. (33) which gives rise to a regular flux.

We have thus established that the extremal limit $M \rightarrow Q$ of the exact relation (8) smoothly connects to the extremal expression $u\left(U_{\mathrm{in}} ; Q\right)$ of Eq. (33). In addition, since the extremal limit applied to the late time expression of (8) given in Eq. (11) is ill-defined, we have demonstrated that the late time limit (i.e. the stationary limit) and the extremal limit do not commute.

This noncommuting character explains why the fluxes obtained using the extremal limit of the exact relation are regular even though those obtained by taking the extremal limit of the stationary fluxes were singular on the horizon. Indeed, it is not difficult to show that the extremal limit of the Schwarzian derivative governing the collapse of a 
NEBH given in Eq. (18) leads to the transients present in Eq. (35) which are necessary to preserve the regularity on the horizon. At fixed $u_{\text {in }}$ and in terms of the parameter $\Delta \equiv$ $\sqrt{M^{2}-Q^{2}}$, when using the coordinate $U_{\text {in }}$ of Eq. (34) to simplify the expression, the Schwarzian derivative of Eq. (18) reads

$$
\begin{aligned}
& -\frac{2 \Delta^{2}}{(M+\Delta)^{2}} \frac{\left[1-\frac{(M-\Delta)^{2}}{(M+\Delta)^{2}} \frac{\left(U_{\text {in }}+2 \Delta\right)^{3}}{\left(U_{\text {in }}-2 \Delta\right)^{3}}\right]}{\left[1-\frac{\Delta}{(M+\Delta)^{2}}\left(U_{\text {in }}+2 \Delta\right)-\frac{(M-\Delta)^{2}}{(M+\Delta)^{2}} \frac{\left(U_{\text {in }}+2 \Delta\right)}{\left(U_{\text {in }}-2 \Delta\right)}\right]^{3}} \\
& +\frac{3 \Delta^{2}}{2(M+\Delta)^{2}} \frac{\left[1-\frac{(M-\Delta)^{2}}{(M+\Delta)^{2}} \frac{\left(U_{\text {in }}+2 \Delta\right)^{2}}{\left(U_{\text {in }}-2 \Delta\right)^{2}}\right]^{2}}{\left[1-\frac{\Delta}{(M+\Delta)^{2}}\left(U_{\text {in }}+2 \Delta\right)-\frac{(M-\Delta)^{2}}{(M+\Delta)^{2}}\left(\frac{\left(U_{\text {in }}+2 \Delta\right)}{\left(U_{\text {in }}-2 \Delta\right)}\right]^{4}\right.} .
\end{aligned}
$$

Taking the extremal limit $\Delta \rightarrow 0$ we are left with

$$
\frac{8 M U_{\text {in }}^{3}}{\left(U_{\text {in }}-2 M\right)^{6}},
$$

which exactly gives the transients of Eq. (35).

As in Eq. (38), the proof relies on the fact that the function $r^{*}(r ; Q, M)$ of Eq. (5) uniformly converges to $r^{*}(r ; M)$ of Eq. (28) in the limit $M \rightarrow Q$, outside the horizon, since the integrand of $r^{*}(r ; Q, M) \quad(=$ $1 / f(r ; M, Q))$ is a differentiable function of $M$ and $Q$.

\section{CONCLUSIONS}

We have seen how a superficial way of treating the extremal limit leads to the conclusion that the stress tensor of EBH is singular on the horizon, unlike what is found for NEBH. We have also shown that the singular behavior results from having assumed the stationarity of the fluxes, which amounts to neglecting transients which are necessary for insuring the regularity on the horizon.

With more details, when starting from the late time expressions of the nonextremal case, one simply misses these transients because they are negligible far away from the hole when compared to the finite Hawking radiation, and on the horizon they only give a finite and regular contribution comparable with that of the steady part that decreases as $\left(r-r_{+}\right)^{2}$. However, for EBH, because of the double zero of the metric function $f(r)$ on the horizon, regularity now requires that the outgoing part of the stress tensor vanishes with four powers of $r-M$. This, together with the fact that the steady part only vanishes with three powers, explains why the transients are not only necessary to preserve the regularity: they must be such that when combined with the steady part, the sum vanishes with four powers. Hence they must vanish with three powers and with a normalization which is independent of the collapse.

We have also demonstrated that when starting with the fluxes of NEBH and applying the extremal limit $(M \rightarrow Q)$ before the late time limit, the regularity is preserved including in the limit, the EBH case. With this we establish that $\mathrm{EBH}$ should not be considered as pathological, since their fluxes are smoothly connected to those of NEBH. Perhaps the most unexpected result is that the transients fluxes that were negligible at late times for NEBH evolve (as $M \rightarrow Q$ ) into the necessary transients which cancel out the divergence of the static energy density on the horizon. What is also unexpected is that their late time behavior is independent of the (regular) collapse one is dealing with: Explicitly, the second term of Eq. (35) behaves as

$$
\lim _{U_{\text {in }} \rightarrow 0}\left\langle T_{u u}\right\rangle_{\text {transients }}=\alpha U_{\text {in }}^{3}+O\left(U_{\text {in }}^{4}\right),
$$

where

$$
\alpha=-\frac{1}{192 \pi M^{5}}
$$

is indeed collapse independent. We have also shown that the regularity of the quantum expectation values follows from the well-defined character of the function $r^{*}(r ; Q, M)$ of Eq. (5) in the extremal limit. This is not surprising since the geometrical optics approximation is exact in two dimensions, i.e., the positive frequency in modes of Eq. (13) are entirely governed by the classical function $U_{\text {in }}(u ; Q, M)$. Therefore the quantum expectation values can only depend on this function and its derivatives [and possibly also on the local metric function $f(r ; M, Q)=$ $\left.d r / d r^{*}\right]$. Since $r^{*}(r ; Q, M)$ is $C^{\infty}$ in $M, Q$, so are $U_{\text {in }}(u ; Q, M)$ and its derivatives.

Finally we discuss the relevance of our conclusions to four-dimensional (or higher than bidimensional) black holes. The fact that for EBH the transients are singular and cancel out the divergence of the static energy density should also be found in any dimension in spite of the presence of "gray-body" factors resulting from the elastic scattering on the static centrifugal barrier. Indeed, the Bogoliubov transformation relating, at fixed angular momentum, the regular in modes (13) to the positive frequency out modes $e^{-i \omega u}$ should possess properties which are independent of the dimensionality, because the latter are singular on the horizon. It is therefore difficult to conceive that the value of a gray-body factor could interfere with the Bogoliubov coefficients in such a way as to give rise to transients which are regular on the horizon. In fact, when assuming that the stress tensor obtained by considering a regular collapse be regular in any dimension, this leaves only two possibilities: either the divergence of the late time transients cancels out that of the static energy density (as is the case in 2D [7] and as found in Refs. [8,9]), or they are both regular. Our reasoning concerning the modes at fixed angular momentum suggests that it is unlikely that the second option be realized. However, this contrasts with the numerical analysis of [21] (see also [22] for spin $1 / 2$ fields) which concluded that the static energy density is regular for $4 \mathrm{D} \mathrm{EBH}$. We are planning to report on this with more details in a future paper. 


\section{ACKNOWLEDGMENTS}

We thank P. Anderson and S. Liberati for interesting comments. A.F. acknowledges the Spanish Grant No. FIS2005-05736-C03-03 and the EU Network MRTN-CT-2004-005104 for financial support.

\section{APPENDIX: 2D STRESS TENSOR}

In this appendix we present the basic properties of the stress energy tensor of a 2D massless field propagating in a stationary metric, since this is all we need in the body of the paper.

We thus consider 2D spacetimes which are static and described by the metric

$$
d s^{2}=-f(r) d t^{2}+f^{-1}(r) d r^{2} .
$$

Introducing the null coordinates $u=t-r^{*}, v=t+r^{*}$ where

$$
r^{*}=\int \frac{d r}{f(r)}
$$

the metric is conformally flat

$$
d s^{2}=-f(r) d u d v .
$$

Therefore, a massless minimally coupled scalar field satisfying the d'Alembert equation will obey, in double null coordinate system, the simplified equation

$$
\partial_{u} \partial_{v} \phi=0 .
$$

Its general solution is thus a sum of a function of only $u$ and one of $v$.

Expanding the field operator in the positive frequency plane waves $e^{-i \omega u}, e^{-i \omega v}$ defines a vacuum state, say $|u, v\rangle$, by annihilation with the destruction operators associated with these positive frequency modes. Two basic properties of the renormalized expectation values of the quantum stress tensor of $\phi$ are used in the text.
First, when $\phi$ propagates in the space-time described by (A3), its stress tensor reads

$$
\left\langle u, v\left|T_{u u}\right| u, v\right\rangle=\left\langle u, v\left|T_{v v}\right| u, v\right\rangle=-\frac{1}{192 \pi}\left(f^{\prime 2}-2 f f^{\prime \prime}\right),
$$

$$
\left\langle u, v\left|T_{u v}\right| u, v\right\rangle=\frac{1}{96 \pi} f f^{\prime \prime},
$$

where a prime indicates derivative with respect to $r$.

The second property follows from the fact that the set of positive frequency modes $\left(e^{-i \omega u}, e^{-i \omega v}\right)$ is not unique, even though it is complete. One could introduce two new null coordinates

$$
U=U(u), \quad V=V(v),
$$

and use these to define a new set of positive frequency modes $\left(e^{-i \lambda U}, e^{-i \lambda V}\right)$. By the same procedure as above, these modes can be used to define another vacuum state, named $|U, V\rangle$. Then the expectation values of the stress tensor in this new state are related to the former one by

$$
\begin{gathered}
\left\langle U, V\left|T_{u u}\right| U, V\right\rangle=\left\langle u, v\left|T_{u u}\right| u, v\right\rangle-\frac{1}{24 \pi}\{U, u\}, \\
\left\langle U, V\left|T_{v v}\right| U, V\right\rangle=\left\langle u, v\left|T_{v v}\right| u, v\right\rangle-\frac{1}{24 \pi}\{V, v\}, \\
\left\langle U, V\left|T_{u v}\right| U, V\right\rangle=\left\langle u, v\left|T_{u v}\right| u, v\right\rangle,
\end{gathered}
$$

where $\{U, u\}$ is the Schwarzian derivative

$$
\{U, u\}=\left(\frac{d U}{d u}\right)^{-1} \frac{d^{3} U}{d u^{3}}-\frac{3}{2}\left(\frac{d U}{d u}\right)^{-2}\left(\frac{d^{2} U}{d u^{2}}\right)^{2},
$$

and similarly for $\{V, v\}$. Equation (A10) is a consequence of the state independence of the trace anomaly.
[1] S. W. Hawking, G. T. Horowitz, and S. F. Ross, Phys. Rev. D 51, 4302 (1995).

[2] C. Teitelboim, Phys. Rev. D 51, 4315 (1995); 52, 6201(E) (1995).

[3] A. Ghosh and P. Mitra, Phys. Lett. B 357, 295 (1995).

[4] S. Das, A. Dasgupta, and P. Ramadevi, Mod. Phys. Lett. A 12, 3067 (1997).

[5] S. Hod, Phys. Rev. D 61, 084018 (2000).

[6] A. Strominger and C. Vafa, Phys. Lett. B 379, 99 (1996).

[7] S. P. Trivedi, Phys. Rev. D 47, 4233 (1993).

[8] V.P. Frolov and A. I. Zelnikov, Phys. Rev. D 35, 3031 (1987).

[9] P. R. Anderson, W. A. Hiscock, and D. A. Samuel, Phys. Rev. D 51, 4337 (1995).
[10] R. Balbinot, S. Fagnocchi, A. Fabbri, S. Farese, and J. Navarro-Salas, Phys. Rev. D 70, 064031 (2004).

[11] S. Fagnocchi and S. Farese, Phys. Rev. D 72, 024015 (2005).

[12] S. A. Fulling, M. Sweeny, and R. M. Wald, Commun. Math. Phys. 63, 257 (1978).

[13] S. Liberati, T. Rothman, and S. Sonego, Phys. Rev. D 62, 024005 (2000).

[14] S. W. Hawking, Commun. Math. Phys. 43, 199 (1975); 46, 206 (1976).

[15] P. G. Grove, Classical Quantum Gravity 7, 1353 (1990).

[16] N.D. Birrell and P.C.W. Davies, Quantum Fields in Curved Space (Cambridge University Press, Cambridge, England, 1982). 
[17] S. M. Christensen and S. A. Fulling, Phys. Rev. D 15, 2088 (1977).

[18] P. C. W. Davies, S. A. Fulling, and W. G. Unruh, Phys. Rev. D 13, 2720 (1976).

[19] D. J. Loranz, W. A. Hiscock, and P. R. Anderson, Phys. Rev. D 52, 4554 (1995).
[20] S. Gao, Phys. Rev. D 68, 044028 (2003).

[21] P. R. Anderson, W. A. Hiscock, and D. J. Loranz, Phys. Rev. Lett. 74, 4365 (1995).

[22] E. D. Carlson, W.H. Hirsch, B. Obermayer, P. R. Anderson, and P. B. Groves, Phys. Rev. Lett. 91, 051301 (2003). 